Research Paper

\title{
Screening Tests Using Serum Tissue Transglutaminase IgA May Facilitate the Identification of Undiagnosed Celiac Disease among Japanese Population
}

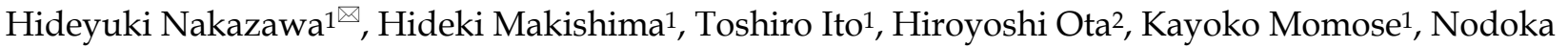
Sekiguchi1, Kaname Yoshizawa ${ }^{3}$, Taiji Akamatsu ${ }^{4}$, Fumihiro Ishida1, 2

1. Department of Internal Medicine, Division of Hematology, Shinshu University School of Medicine, 3-1-1 Asahi Matsumoto, Japan 390-8621;

2. Department of Biomedical Laboratory Sciences, School of Health Sciences, Shinshu University School of Medicine, 3-1-1 Asahi Matsumoto, Japan 390-8621:

3. Department of Internal Medicine, Division of Gastroenterology and Hepatology, Shinshu University School of Medicine, 3-1-1 Asahi Matsumoto, Japan 390-8621;

4. Department of Endoscopy, Shinshu University Hospital, 3-1-1 Asahi Matsumoto, Japan 390-8621

$\square$ Corresponding author: Hideyuki Nakazawa, MD; Department of Internal Medicine, Division of Hematology, Shinshu University School of Medicine, 3-1-1 Asahi Matsumoto, Japan 390-8621; Tel;+81-263-37-2634, Fax+81-263-32-9412, E-mail hnaka@shinshu-u.ac.jp

() Ivyspring International Publisher. This is an open-access article distributed under the terms of the Creative Commons License (http://creativecommons.org/ licenses/by-nc-nd/3.0/). Reproduction is permitted for personal, noncommercial use, provided that the article is in whole, unmodified, and properly cited.

Received: 2014.02.17; Accepted: 2014.05.02; Published: 2014.06.03

\begin{abstract}
The prevalence of celiac disease (CD) among Japanese population has been unknown, whereas it has been increasingly recognized in the US and in the European countries. The aim of the present study is to identify possible cases with $C D$ among Japanese population and clarify the relevance of screening for the disease.

We conducted a serologic screening for the disease among 710 Japanese patients and 239 healthy volunteers at a local tertiary teaching hospital, using an anti-tissue transglutaminase lgA (TTG-IgA) test, and histological examination of the small intestines from the TTG-IgA positive subjects.

There were no TTG-IgA positive sera among the healthy volunteers. Twenty of the patients (2.8\%), including eight with malignant lymphoma, were tested positive for TTG-IgA. The histological examination of the eleven patients among those with positive TTG-IgA, seven showed villous atrophy and partial lymphocytes infiltration in the mucosa, which could be compatible to mucosal changes observed in CD. Five of them had non-Hodgkin lymphoma in the gastrointestinal tracts.
\end{abstract}

Serologic tests using TTG-IgA might be relevant to screen for those with undiagnosed CD among Japanese population.

Key words: celiac disease, anti-tissue transglutaminase IgA, screening, malignant lymphoma

\section{BACKGROUND}

Celiac disease $(\mathrm{CD})$ is an autoimmune-type of gastrointestinal disorder, triggered by gluten-containing grains [1]. Its clinical presentation has been found to be highly variable, ranging from typical diarrheal symptoms to the conditions that can affect any organ systems, such as iron deficiency anemia, osteoporosis, ataxia, type-I diabetes, or dermatitis herpetiformis [2, 3]. Even silent cases of the disease have been increasingly recognized [4]. It is also known to be associated with an increased risk for non-Hodgkin lymphoma, especially of the T-cell origin and primarily localized in gastrointestinal tract, such as enteropathy-associated T-cell lymphoma (EATL) $[5,6]$. Controlling of CD with strict adherence to the gluten free diet is suggested to be the only pos- 
sible method of prevention from the lymphoma development [6]. However, many CD cases are known to be left undiagnosed and may be carrying a risk of long-term complications, including malignant lymphoma [7].

With the advent of multiple serologic tests for $\mathrm{CD}$, a substantial number of undiagnosed cases have been increasingly recognized, yielding the prevalence as approximately $1 \%$ among the Caucasian population in the US and the Europe [1,8]. Screening programs have facilitated identification of undiagnosed $\mathrm{CD}$ in different parts of the world [9-11]. We conducted serologic tests among Japanese subjects and examined the possible diagnosis of $\mathrm{CD}$ among this population.

\section{METHODS}

\section{Patients and healthy donors}

We recruited 710 patients and 239 healthy individuals for the screening, between 1980 and 2007, at Shinshu University School of Medicine and its university hospital, a local tertiary teaching hospital, in Matsumoto, Japan. The clinical profiles of the patients were reviewed in their charts, and their main diagnoses were identified according to their clinical problems for which the each patient had needed medical attentions most. Their diagnoses comprised malignant lymphoma $(n=254)$, gastric and/or duodenal ulcer $(n=59)$, acute or chronic leukemia $(n=57)$, viral hepatitis and/or liver cirrhosis $(n=28)$, myelodysplastic syndrome $(n=25)$, multiple myeloma $(n=22)$, carcinoma in gastrointestinal tracts $(n=16)$, or inflammatory bowel disease $(n=12)$. The demographic comparison between the patients and the healthy volunteers showed that the former was higher in age $(p<0.0001)$ and the latter with female predominance $(p<0.001)$. The serum samples had been obtained according to the Declaration of Helsinki and stored at $-20^{\circ} \mathrm{C}$ until studied. This study protocol was approved by the institutional review board of Shinshu University School of Medicine.

\section{Measurement of anti-tissue transglutaminase IgA antibody (TTG-IgA) with enzyme-linked immunosorbent assay (ELISA)}

The serum samples were assayed using ORG 540A Anti-Tissue-Transglutaminase IgA (American Research Products, Belmont, MA) in accordance with the manufacturer's protocol in a blinded fashion. In short, the diluted serum samples were incubated in the microplate wells coated with human recombinant tissue transglutaminase for 30 minutes at room temperature. Following 15-minute incubation with enzyme conjugate, containing polyclonal rabbit an- ti-human IgA labeled with horseradish peroxidase, Tetramethylbenzidine substrate solution was dispensed for another 15 minutes. After reaction was stopped with hydrochloric acid, the optical density at $450 \mathrm{~nm}$ of each well was read with bi-chromatic measurement with a reference at $650 \mathrm{~nm}$. Each serum samples, calibrators, and controls were processed in a duplicated manner. The upper limit of a normal range of serum TTG-IgA for healthy control group was presumably adapted from the manufacturer's guideline, at the cut-off value of $10.0 \mathrm{U} / \mathrm{ml}$. The lower detection limit for TTG-IgA was $1.0 \mathrm{U} / \mathrm{ml}$. A previous review about diagnostic accuracy of serologic tests using human recombinant TTG-IgA revealed $98.1 \%$ of sensitivity and $98.0 \%$ of specificity among Caucasian adult population [12].

\section{Histological examination of small bowels}

Upper gastrointestinal endoscopy and multiple duodenal biopsies were performed for the individuals with a positive result for TTG-IgA. When the endoscopic examination was not performed, we looked for the biopsied samples or surgical specimen of small intestine obtained during surgery in the past, if any. Formalin-fixed biopsy specimens were stained with hematoxylin and eosin and were studied under a light microscopy, searching for the architectural changes in intestinal mucosa, such as increased intraepithelial lymphocytes, crypt hyperplasia, and villous atrophy. The Marsh-Oberhuber grading system was applied for pathological diagnosis of CD. In short, the presence of immune response in the epithelium and the degree of architectural changes in the mucosa was assessed and categorized in the grading manner from type 0 to type 3 [13].

\section{Statistical analysis}

The correlations between TTG-IgA positivity and the age and sex of the subjects, and their disease status, such as being affected with malignant lymphoma and having gastrointestinal tract lymphoma, were analyzed statistically, using Mann-Whitney U test or Chi square test (StatView for Windows Version $5.0)$.

\section{RESULTS}

\section{Serological screening}

Figure 1 shows a scatter diagram of TTG-IgA values of the enrolled subjects. The mean TTG-IgA value of the healthy group was $0.7 \mathrm{U} / \mathrm{ml}(2 \mathrm{SD}=1.7$ $\mathrm{U} / \mathrm{ml}$ ), whereas that of the patient group was 2.5 $\mathrm{U} / \mathrm{ml}(2 \mathrm{SD}=24.4 \mathrm{U} / \mathrm{ml}$, maximum value $294 \mathrm{U} / \mathrm{ml})$. Twenty of the patient group $(2.8 \%)$ tested positive for TTG-IgA, whereas none of the healthy volunteer group were positive $(p<0.01)$. Compared with the 


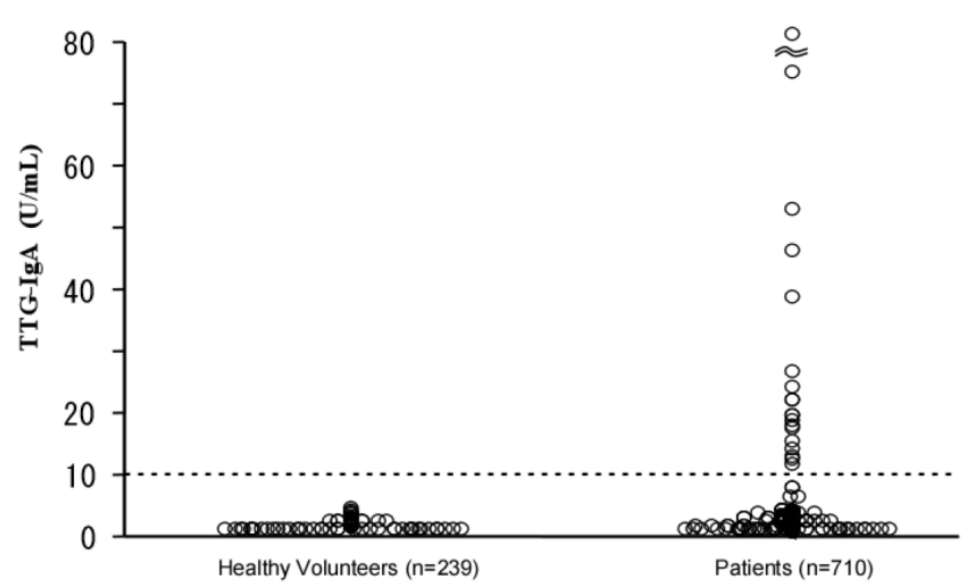

Figure I. Scatter diagram of serum anti-tissue transglutaminase $\lg A$ antibody (TTG-IgA) in the enrolled subjects.

TTG-IgA negative patients, the positive patients were higher in age $(\mathrm{p}<0.05)$, though sexual predominance was not observed.

The reasons of the hospital visits of the 20 patients with TTG-IgA positive result are either malignant lymphoma $(n=8)$, gastritis $(n=2)$, multiple myeloma, leukemia, cytopenia, Castleman's disease, colon polyps, type-II diabetes mellitus, osteoporosis, liver cirrhosis, or Budd-Chiari syndrome. The histology of the eight malignant lymphomas entailed six with B-cell type and two with T-cell type. Neither of the T-cell lymphomas was enteropathy associated T-cell lymphoma (EATL). Six of the eight lymphoma patients with positive TTG-IgA had gastrointestinal involvement of malignant lymphoma at presentation, whereas 87 of the 246 lymphoma patients with negative TTG-IgA test had gastrointestinal disease $(\mathrm{p}=0.055)$.

\section{Pathological findings of small bowel mucosa}

Of the 20 patients with positive TTG-IgA values, duodenal biopsy or small bowel mucosal specimen was available in eleven patients. The seven of the eleven patients exhibited villous atrophy and partial infiltration of intraepithelial lymphocytes, which could be in accordance with the type 3 lesions in the Marsh-Oberhuber grading system.

\section{Clinical profiles of the seven patients with both positive TTG-IgA and the pathological findings (Table 1)}

All seven were Japanese natives with the median age of 54 years (range; 27-74 years). Six of the seven were male. There was no documentation of symptomatic family history. The five of the seven patients had malignant lymphoma, including mucosa-associated lymphoid tissue (MALT) lymphoma $(n=2)$, diffuse large B-cell lymphoma (DLBCL) $(n=1)$, and peripheral T-cell lymphoma, unspecified (PTCL-u) $(n=1)$. The other presented both DLBCL and PTCL-u at different times during the 28 months of his clinical course (patient \#2), which was the case reported previously [14, 15]. All five cases had lymphoma lesions in the gastrointestinal tract.

Of the seven patients, three died from the malignant lymphoma and another from anasarca. Another is alive on gluten-free diet with complete response of the malignancies. One of the remaining two is alive with gluten-free diet and the other has lost contact.

Table I. The seven patients with positive TTG-IgA and pertinent mucosal changes

\begin{tabular}{|c|c|c|c|c|c|c|c|c|c|}
\hline $\begin{array}{c}\text { Patient index } \\
\text { number }\end{array}$ & Sex / Age, y & $\begin{array}{c}\text { Reasons of } \\
\text { Hospital Visits } \\
\end{array}$ & Diarrhea & $\begin{array}{c}\text { Clinical Improvement } \\
\text { with GFD }\end{array}$ & $\begin{array}{c}\text { Iron Deficiency } \\
\text { Anemia }\end{array}$ & $\begin{array}{l}\text { TTG-lgA } \\
(\mathrm{U} / \mathrm{ml}) \\
\end{array}$ & $\begin{array}{c}\lg A \\
(\mathrm{mg} / \mathrm{dl})\end{array}$ & $\begin{array}{c}\text { family history of } \\
C D \\
\end{array}$ & Outcome \\
\hline$\# 2$ & M54 & $\mathrm{ML}^{*}$ & + & + & - & 75.9 & 261 & - & alive \\
\hline$\# 3$ & M65 & $\mathrm{ML}$ & + & + & + & 523 & 650 & $t^{* *}$ & $28 \mathrm{M}^{[]}$dead \\
\hline & & & & & & & & & \\
\hline$\# 4$ & M32 & $\mathrm{ML}$ & + & $N A^{\#}$ & - & 38.5 & 221 & - & $39 \mathrm{M}$ dead \\
\hline$\# 9$ & M54 & $\mathrm{ML}$ & - & NA & - & 12.5 & 785 & - & $70 \mathrm{M}$ dead \\
\hline & & & & & & & & & \\
\hline$\# 10$ & M67 & $\mathrm{ML}$ & + & NA & - & 10.5 & 1640 & - & $36 \mathrm{M}$ dead \\
\hline$\# 13$ & $F / 27$ & Budd-C & - & NA & - & 21.7 & 201 & NA & NA \\
\hline \# 17 & N70 & DM & - & NA & - & 17.2 & 748 & NA & alive \\
\hline & & & & & & & & & \\
\hline \multicolumn{10}{|c|}{ GFD: gluten free diet; "ML: malignant lymphoma; Budd-C: Budd-chiari syndrome; DM: diabetes mellitus type Il; } \\
\hline \pm : seropositive & ologically ne & family; "NA: no & available; & 28M: 28 months. & & & & & \\
\hline
\end{tabular}




\section{DISCUSSION}

Since the advent of serologic screening tools and growing awareness of the disease, a substantial number of undiagnosed cases have been increasingly recognized in different parts of the world, and the epidemiological interests seem to prevail even in East Asia, where the prevalence of the disease has been presumed to be significantly low [10, 16-19]. This is the first and largest case finding study for the possible diagnosis of $C D$ among Japanese population. Our screening program among 710 patients identified seven cases $(0.98 \%)$ with both positive TTG-IgA and the pertinent mucosal changes, compatible with celiac disease. The present study would shed light on the discussion of possible diagnosis of celiac disease among East Asian population.

The definitive diagnosis of celiac disease in the present study, however, could hardly be verified for several reasons. First, five of the seven patients with possible diagnosis of $\mathrm{CD}$ had been concomitantly diagnosed with malignant lymphoma, including four cases with B-cell lymphoma. Enteropathy-type T-cell lymphoma (EATL) was not observed. The pathological examinations of their small bowel mucosa were conducted either after initiation of chemotherapy $(n=2)$, during non-CR period $(n=1)$, or after radiation therapy $(n=1)$. By definition, the diagnosis of celiac disease among those with EATL may be straightforward. However, it may be inherently challenging to make a diagnosis of celiac disease, when the patients are found to have non-EATL lymphoma concomitantly without the typical symptoms nor known family history of celiac disease, like the present cases. The intestinal mucosal damage could be induced by chemotherapy and radiotherapy, which might be confused with the mucosal atrophy caused by celiac disease. Also, the villous atrophy is shown to coexist with intestinal lymphoma [1].

The lack of HLA screening test would be another shortcoming of the present study. Mounting evidence suggests that more than $99 \%$ of CD patients in the US and Europe share HLA DQ2 and/or HLA DQ8 alleles, and negative predictive value is more than $95 \%$ [20]. However, some single CD patient may remain negative for both HLA-DQ2 and -DQ8 haplotypes $[20,21]$. Kaukinen et al emphasize that a negative HLA finding does not rule out CD when the clinical context shows otherwise, though such condition may be rare in Europe [20]. Meanwhile, different HLA typings and genetic variation are known to confer same phenotype in the diseases, such as in autoimmune hepatitis, for example [22]. Although our data is too small to indicate alternative HLA typings, the negative predictive value of HLA typings in East Asia should be decided independently.

Large proportion of our subjects had malignant lymphoma and $40 \%$ of TTG-IgA positive patients were lymphoma patients. This overexpression of malignant lymphoma is partly due to our index case that had gastrointestinal lymphoma associated with $C D$ [14]. With the scarce data at hand on the actual prevalence of celiac disease among non-Caucasian population, our initial interests were focused on a case finding study through recruiting pertinent patients with symptoms and/or diseases related to those of the index case. Again, it must be noted that the identical mucosal changes found in the present study could be observed those with malignant lymphoma or the therapies against lymphomas, which is the inevitable drawback.

Meanwhile, the increased risks for non-Hodgkin lymphoma other than EATL among CD patients have been widely studied [23, 24], and the relationship has not been examined among non-Caucasoid population. Despite of our selection bias, it could be speculated that our results may support the possible increased prevalence of CD-associated B-cell lymphoma. Furthermore, it is not yet clear whether the gastrointestinal tract disease may be increased as the primary location of non-EATL lymphoma in CD patients [25]. Our result, however, showed the non-significant increase in TTG-IgA positivity among the patients with gastrointestinal lymphoma. Conversely, it could be speculated that such lymphomatous infiltration might have influenced antigenicity to produce TTG-IgA.

Baldas et al showed an age-related increase in TTG-IgA titers among the non-celiac general population, suggesting the cutoff points for serological screening to be carefully evaluated [26]. Unlike their result, the age-dependent increase in TTG-IgA titer was found to be minimal in our subjects. Although the influence of age on TTG-IgA titers should also be taken into account at the interpretation of this study results, the validity of our cutoff value is beyond the scope of this study.

Another diagnostic pitfall of our present study may be the lack of longitudinal observation with strict gluten free diet and gluten challenge. Increasing application of serology for its diagnosis seem to have encouraged a shift from three-biopsy diagnostic algorism to one-biopsy algorism, leading possible cases of overdiagnosis even in US [27]. Confusion in the definition of strict gluten free diet may also be the case in longitudinal observation [28]. The retrospective nature of the present study is hardly up to these points.

Our extensive literature search has revealed possible cases with underdiagnosed celiac disease and dermatitis herpetiformis in Japan, the latter of which is known to be pathognomonic for CD among Cauca- 
sian population [17, 29-33]. They should encourage the discussion of $\mathrm{CD}$ among Japanese population, and our study result might show the relevance of serologic tests as screening for the disease in the population.

In conclusion, serologic tests using TTG-IgA might be relevant to identify those with undiagnosed $\mathrm{CD}$ among Japanese population.

\section{ACKNOWLEDGEMENTS}

The authors are indebted to Dr Kiyoshi Kitano, Matsumoto Medical Center, Matsumoto, Japan, for his support in preparing the manuscript.

\section{COMPETING INTERESTS}

The authors have declared that no competing interest exists.

\section{REFERENCES}

1. Green PH, Cellier C. Celiac disease. The New England journal of medicine. 2007; 357: 1731-43

2. Farrell RJ, Kelly CP. Celiac sprue. The New England journal of medicine. 2002; 346: $180-8$.

3. Dewar DH, Ciclitira PJ. Clinical features and diagnosis of celiac disease. Gastroenterology. 2005; 128: S19-S24.

4. Bottaro G, Cataldo F, Rotolo N, Spina M, Corazza GR. The clinical pattern of subclinical/silent celiac disease: an analysis on 1026 consecutive cases. The American journal of gastroenterology. 1999; 94: 691-6.

5. Catassi C, Bearzi I, Holmes GK. Association of celiac disease and intestinal lymphomas and other cancers. Gastroenterology. 2005; 128: S79-86.

6. Catassi C, Bearzi I, Holmes GKT. Association of celiac disease and intestinal lymphomas and other cancers. Gastroenterology. 2005; 128: S79-S86.

7. Green PH. The many faces of celiac disease: clinical presentation of celiac disease in the adult population. Gastroenterology. 2005; 128: S74-8.

8. Fasano A, Berti I, Gerarduzzi T, Not T, Colletti RB, Drago S, et al. Prevalence of celiac disease in at-risk and not-at-risk groups in the United States: a large multicenter study. Archives of internal medicine. 2003; 163: 286-92.

9. Cummins AG, Roberts-Thomson IC. Prevalence of celiac disease in the Asia-Pacific region. Journal of gastroenterology and hepatology. 2009; 24: 1347-51.

10. Makharia GK, Verma AK, Amarchand R, Bhatnagar S, Das P, Goswami A, et al. Prevalence of celiac disease in the northern part of India: a community based study. Journal of gastroenterology and hepatology. 2011; 26: 894-900.

11. Gandolfi L, Pratesi R, Cordoba JC, Tauil PL, Gasparin M, Catassi C. Prevalence of celiac disease among blood donors in Brazil. The American journal of gastroenterology. 2000; 95: 689-92.

12. Rostom A, Dubé C, Cranney A, Saloojee N, Sy R, Garritty C, et al. The diagnostic accuracy of serologic tests for celiac disease: A systematic review. Gastroenterology. 2005; 128: S38-S46.

13. Dickson BC, Streutker CJ, Chetty R. Coeliac disease: an update for pathologists. Journal of clinical pathology. 2006; 59: 1008-16

14. Makishima $H$, Ito $T$, Kodama $R$, Asano $N$, Nakazawa $H$, Hirabayashi $K$, et al. Intestinal diffuse large B-cell lymphoma associated with celiac disease: a Japanese case. International journal of hematology. 2006; 83: 63-5.

15. Makishima H, Komiyama $\mathrm{Y}$, Asano N, Momose K, Nakamura S, Ishida F. Peripheral T-cell lymphoma following diffuse large B-cell lymphoma associated with celiac disease. Intern Med. 2008; 47: 295-8.

16. Wu J, Xia B, von Blomberg BM, Zhao C, Yang XW, Crusius JB, et al. Coeliac disease: emerging in China? Gut. 2010; 59: 418-9.

17. Ohata C, Ishii N, Hamada T, Shimomura Y, Niizeki H, Dainichi T, et al. Distinct characteristics in Japanese dermatitis herpetiformis: a review of all 91 Japanese patients over the last 35 years. Clinical \& developmental immunology. 2012; 2012: 562168.

18. Jiang LL, Zhang BL, Liu YS. Is adult celiac disease really uncommon in Chinese? Journal of Zhejiang University Science B. 2009; 10: 168-71.

19. Watanabe $\mathrm{C}$, Komoto $\mathrm{S}$, Hokari $\mathrm{R}$, Kurihara $\mathrm{C}$, Okada $\mathrm{Y}, \mathrm{Hozumi} \mathrm{H}$, et al Prevalence of serum celiac antibody in patients with IBD in Japan. Journal of gastroenterology. 2013..

20. Kaukinen K, Partanen J, Maki M, Collin P. HLA-DQ typing in the diagnosis of celiac disease. The American journal of gastroenterology. 2002; 97: 695-9.

21. Karell K, Louka AS, Moodie SJ, Ascher H, Clot F, Greco L, et al. Hla types in celiac disease patients not carrying the DQA1*05-DQB1*02 (DQ2) heterodimer: results from the european genetics cluster on celiac disease. Human Immunology. 2003; 64: 469-77.
22. Seki $\mathrm{T}$, Ota $\mathrm{M}$, Furuta $\mathrm{S}$, Fukushima $\mathrm{H}$, Kondo $\mathrm{T}$, Hino $\mathrm{K}$, et al. HLA class II molecules and autoimmune hepatitis susceptibility in Japanese patients. Gastroenterology. 1992; 103: 1041-7.

23. Catassi C, Fabiani E, Corrao G, Barbato M, De Renzo A, Carella AM, et al. Risk of non-Hodgkin lymphoma in celiac disease. JAMA : the journal of the American Medical Association. 2002; 287: 1413-9.

24. Green PH, Jabri B. Coeliac disease. Lancet. 2003; 362: 383-91.

25. Smedby KE, Baecklund E, Askling J. Malignant lymphomas in autoimmunity and inflammation: a review of risks, risk factors, and lymphoma characteristics. Cancer epidemiology, biomarkers \& prevention : a publication of the American Association for Cancer Research, cosponsored by the American Society of Preventive Oncology. 2006; 15: 2069-77.

26. Baldas V, Not T, Tommasini A, Ansaldi F, Demarini S, Sblattero D, et al. Anti-transglutaminase antibodies and age. Clinical chemistry. 2004; 50: 1856-60.

27. Catassi C, Fasano A. Is this really celiac disease? Pitfalls in diagnosis. Current gastroenterology reports. 2008; 10: 466-72.

28. Kupper C. Dietary guidelines and implementation for celiac disease. Gastroenterology. 2005; 128: S121-7.

29. Shibahara M, Nanko H, Shimizu M, Kanda N, Kubo M, Ikeda M, et al. Dermatitis herpetiformis in Japan: an update. Dermatology. 2002; 204: 37-42.

30. Kataoka I, Arima F, Nishimoto J, Watanabe T, Kobayashi Y, Tamura R, et al. Enteropathy-type T-cell lymphoma showing repeated small bowel rupture and refractoriness to chemotherapy: a case report. Japanese journal of clinical oncology. 2002; 32: 546-9.

31. Yasuoka H, Masuo T, Hashimoto K, Sato K, Okada S, Kusano M, et al. Enteropathy-type T-cell Lymphoma that was Pathologically Diagnosed as Celiac Disease Internal Medicine. 2007; 46: 1219-24.

32. Asano Y, Makino T, Ishida W, Furuichi M, Shimizu T. Detection of antibodies to epidermal transglutaminase but not tissue transglutaminase in Japanese patients with dermatitis herpetiformis. The British journal of dermatology. 2011; 164: 883-4.

33. Amo Y, Tanei R, Tanabe K, Katsuoka K. Dermatitis herpetiformis in a Japanese patient with anaplastic large cell lymphoma. The Journal of dermatology. 2000; 27: 533-6. 